Pacific

Journal of

Mathematics

ON THE COMPOSITION OF A PRIME TRANSCENDENTAL FUNCTION AND A PRIME POLYNOMIAL

Tuen-Wai Ng And Chung-Chun Yang

Volume 193 No. 1

March 2000 


\title{
ON THE COMPOSITION OF A PRIME TRANSCENDENTAL FUNCTION AND A PRIME POLYNOMIAL
}

\author{
Tuen-Wai Ng And Chung-Chun Yang
}

Let $f, g$ be transcendental entire functions and $p, q$ be nonlinear polynomials with $\operatorname{deg} p \neq 3,6$. Suppose that $f$ and $p$ are prime and $f(p(z))=g(q(z))$, then $f=g \circ L$ and $p=L^{-1} \circ q$, where $L$ is a linear polynomial. Similar results for $p(f(z))=q(g(z))$ are also obtained.

\section{Introduction and Main Results.}

A meromorphic function $F(z)$ is said to has a factorization with left factor $f$ and right factor $g$ provided

$$
F(z)=f(g(z))
$$

where $f$ is meromorphic and $g$ is entire ( $g$ may be meromorphic when $f$ is rational). A nonlinear meromorphic function $F(z)$ is called prime (pseudo prime) if every factorization of form (1) implies that either $f$ is bilinear or $g$ is linear (either $f$ is rational or $g$ is a polynomial). Clearly, a prime function is an analogy of a prime number. Over the past thirty years, many classes of prime or pseudo-prime functions have been obtained (see [2]).

As an analogue of the unique factorizability of natural numbers, one can also define that concept for entire functions. Suppose an entire function $F$ has two factorizations $f_{1} \circ f_{2} \circ \cdots \circ f_{m}(z)$ and $g_{1} \circ g_{2} \circ \cdots \circ g_{n}(z)$ into nonlinear entire factors. If $m=n$ and if there exist linear polynomials $L_{j}$ $(j=1,2,3, \ldots, n-1)$ such that the relations

$$
f_{1}(z)=g_{1} \circ L_{1}^{-1}, \quad f_{2}(z)=L_{1} \circ g_{2} \circ L_{2}^{-1}, \quad \ldots \ldots . \quad, \quad f_{n}(z)=L_{n-1} \circ g_{n}(z)
$$

hold simultaneously, then the two factorizations are called equivalent. If any two factorizations of $F(z)$ into nonlinear, prime entire factors are equivalent to each other, then $F$ is called uniquely factorizable in entire sense.

As far as just polynomial factors are concerned, it is easy to exhibit functions which are not uniquely factorizable in entire sense, for instance, $z^{3} \circ z^{2}=z^{2} \circ z^{3}$.

Therefore, the following question is not without interest. 
Problem (A). Suppose $f$ and $g$ are prime entire functions and one of them is transcendental, will $F(z)=f \circ g(z)$ be uniquely factorizable in entire sense?

Counter-example. Take $f(z)=z^{2}, g(z)=z e^{z^{2}}, f_{1}(z)=z e^{2 z}$ and $g_{1}(z)=$ $z^{2}$. All of them are prime functions (see [2]) and $f \circ g=f_{1} \circ g_{1}$ are two nonequivalent factorizations of $z^{2} e^{2 z^{2}}$.

In this paper, we shall consider the following problems. Let $f$ and $p$ be two prime entire functions where $f$ is transcendental and $p$ is a polynomial. Suppose that $f \circ p=g \circ q$ or $p \circ f=q \circ g$. Under what conditions on the entire functions $g, q$ will these factorizations be equivalent?

From the above counterexample, it is clear that two factorizations of a function $F=h \circ k=h_{1} \circ k_{1}$ may not be equivalent. Therefore, we need to have some further assumptions on these factors $h, h_{1}, k$ and $k_{1}$.

With this in mind, we have come up with the following results. The functions $f, g, p$ and $q$ considered below are all entire and nonlinear.

Theorem 1. Let $f, p$ be two non-periodic prime entire functions and $p$ be a polynomial. Suppose that $p \circ f=q \circ g$ and both $f, g$ are transcendental. Then $p=q \circ L^{-1}$ and $f=L \circ g$, where $L$ is a linear polynomial.

Theorem 2. Let $f, p$ be two prime entire functions and $f$ be transcendental. Suppose that $p \circ f=q \circ g$ and both $p, q$ are polynomials. Then $p=q \circ L^{-1}$ and $f=L \circ g$, where $L$ is a linear polynomial.

Theorem 3. Let $f$, $p$ be two prime entire functions and $f$ be transcendental. Suppose that $f \circ p=g \circ q$ and both $p, q$ are polynomials with $\operatorname{deg} p \neq 3,6$. Then $f=g \circ L$ and $p=L^{-1} \circ q$, where $L$ is a linear polynomial.

Theorem 1, 2 and 3 deal with the relationships between polynomials $p$ and $q$, transcendental functions $f$ and $g$ when we have factorizations of the form $p \circ f=q \circ g$ or $f \circ p=g \circ q$. It is natural to investigate the case $f \circ p=q \circ g$.

Theorem 4. Let $f$ and $g$ be two transcendental entire functions, $p$ and $q$ be two nonlinear polynomials with degree $n$ and $m$ respectively. If $f \circ p=q \circ g$ and $p$ is not a right factor of $g$, then $\operatorname{deg} p \leq \operatorname{deg} q$. In particular, the conclusion is true when $g$ is prime.

Remark 1. Let $f(z)=e^{z}, g(z)=e^{\frac{z^{3}}{2}}, p(z)=z^{3}$ and $q(z)=z^{2}$. Then $f \circ p=q \circ g$ and $\operatorname{deg} p>\operatorname{deg} q$. Therefore, the condition that $p$ is not a right factor of $g$ is essential.

Definition 1. Let $F(z)$ be an nonconstant entire function. An entire function $g(z)$ is a generalized right factor of $F$ (denoted by $g \leq F$ ) if there exists a function $f$, which is analytic on the image of $g$, such that $F=f \circ g$. If such $f$ is entire, $g$ will be a right factor of $F$ (denoted by $g \mid F$ ). 
Definition 2. If $h \leq f$ and $h \leq g$, we say that $h$ is a generalized common right factor of $f$ and $g$. If $g \leq F$ and $f \leq F$, we say that $F$ is a generalized common left multiple of $f$ and $g$.

The existence and uniqueness problems of the greatest generalized common right factor and the least generalized common left multiple for a given pair of entire functions were solved by A. Eremenko and L.A. Rubel as follows.

Lemma 1 ([4]). Any pair of non-constant entire functions has (up to a linear factor) a unique greatest generalized common right factor $h$, greatest in the sense that any generalized common right factor of $f$ and $g$ is a generalized right factor of $h$.

Lemma 2 ([4]). Suppose that $f$ and $g$ have a generalized common left multiple. Then $f$ and $g$ have (up to a linear factor) a unique least generalized common left multiple $F$, least in the sense that $F$ is a generalized right factor of any generalized common left multiple of $f$ and $g$.

The proof of Theorem 1 is mainly based on the following lemma.

Lemma 3 ([10]). Let $f$ and $g$ be two entire functions. Suppose that there exist two nonconstant complex functions $k$ and $R$ such that $F=R \circ f=k \circ g$ is meromorphic. If $g$ is transcendental and $R$ is rational, then there exists a transcendental entire function $h$ satisfying $h \leq f$ and $h \leq g$.

Proof of Theorem 1. By Lemma 3, there exists a transcendental entire function $h$ satisfying $h \leq f$ and $h \leq g$. Hence, $f=h_{1} \circ h$ and $g=h_{2} \circ h$, where $h_{1}, h_{2}$ are analytic on the image of $h$. If the image of $h$ is $\mathbf{C}-\{a\}$, then $h=a+e^{k}$ for some entire function $k$. Without loss of generality, we may assume $a=0$ so that $f(z)=h_{1}\left(e^{w}\right) \circ k(z)$. The primeness of $f$ will force $k$ to be linear. This contradicts the assumption that $f$ is not a periodic function. So the image of $h$ must be the whole plane. This implies that both $h_{1}, h_{2}$ are entire and $p \circ h_{1}=q \circ h_{2}$ on $\mathbf{C}$. Since $f=h_{1} \circ h$ is prime, $h_{1}$ must be linear. From $p \circ h_{1}=q \circ h_{2}, h_{2}$ must also be linear as $p$ is prime. Take $L=h_{1} \circ h_{2}^{-1}$ and we are done.

The proof of Theorem 2 is similar, we simply apply Lemma 4 below instead of Lemma 3.

Lemma $4([6])$. Let $f$ and $g$ be two entire functions. Suppose that there exist two nonconstant polynomials $p$ and $q$ such that $p \circ f(z)=q \circ g(z)$. Then there exist an entire function $h$ and rational functions $U(z)$ and $V(z)$ such that

$$
f(z)=U \circ h(z), \quad g(z)=V \circ h(z) .
$$

To prove Theorem 4, we need the following lemma which can be used to prove Lemma 3. 
Lemma 5 ([10]). Let $f$ and $g$ be two entire functions. Suppose that there exist two nonconstant functions $h_{1}$ and $h_{2}$ so that $F=h_{1}(f(z))=h_{2}(g(z))$ and $F$ is meromorphic. Suppose further that there exist $k \geq 2$ distinct points $z_{1}, \ldots . ., z_{k}$ such that $F^{\prime}\left(z_{i}\right) \neq 0, \infty$ for all $i$ and

$$
\left\{\begin{array}{l}
f\left(z_{1}\right)=f\left(z_{2}\right)=\ldots . . f\left(z_{k}\right) \\
g\left(z_{1}\right)=g\left(z_{2}\right)=\ldots \ldots g\left(z_{k}\right) .
\end{array}\right.
$$

Then, there exists an entire function $h(z)$ (independent of $k$ and $z_{i}^{\prime} s$ ) with $h \leq f, h \leq g$ and $h\left(z_{1}\right)=h\left(z_{i}\right)$ for all $2 \leq i \leq k$.

Proof of Theorem 4. By Lemma 1, there exists a generalized greatest common right factor $k$ of $p$ and $g$. Since, $p$ is a polynomial, $k$ is actually the greatest common right factor of $p$ and $g$. Let $p_{1}$ and $g_{1}$ be entire functions such that $p=p_{1} \circ k$ and $g=g_{1} \circ k$. Hence, $f \circ p_{1}=q \circ g_{1}$ on $\mathbf{C}$ and $p_{1}, g_{1}$ do not have any nonlinear common right factor. $p_{1}$ is nonlinear as $p$ is not a right factor of $g$. If we can show that $\operatorname{deg} p_{1} \leq \operatorname{deg} q_{1}$, then $\operatorname{deg} p \leq \operatorname{deg} q$. Therefore, we may assume that $p$ and $g$ do not have any nonlinear common right factor. Suppose that $n>m$. Define $E=\left\{p(z) \mid F^{\prime}(z)=0\right\}$, where $F=f \circ p$. Then $E$ is a countable set. Therefore, we can choose $A \in \mathbf{C}-E$ so that the equation $p(z)=A$ has $n \geq 2$ distinct roots $z_{1}, \ldots, z_{n}$. Since $f(A)=f\left(p\left(z_{i}\right)\right)=q\left(g\left(z_{i}\right)\right), g\left(z_{i}\right)$ are roots of the equation $q(z)=f(A)$ which has at most $m$ roots. $n>m$ implies that there exist two distinct roots $z_{i}, z_{j}$ such that $g\left(z_{i}\right)=g\left(z_{j}\right)$. Note that $p\left(z_{i}\right)=p\left(z_{j}\right)=A$ and $F^{\prime}\left(z_{i}\right), F^{\prime}\left(z_{j}\right) \neq 0$. By Lemma 5 , there exists an entire function $h$ with $h \leq p, h \leq g$ and $h\left(z_{i}\right)=h\left(z_{j}\right)$. Clearly $h$ is a polynomial. Hence, there exists a nonlinear $h$ such that $h \mid p$ and $h \mid g$. This is impossible and we must have $n \leq m$.

In Theorem 3 , we only assume that $p$ and $q$ are polynomials. If we further restrict $p$ and $q$ to have $\operatorname{deg} p=\operatorname{deg} q \geq 3$, then the conclusion of Theorem 3 can be drawn directly from the following lemma.

Lemma 6 ([5]). Let $p$ and $q$ be two polynomials with the same degree. Suppose there exist entire functions $f$ and $g$ such that $f \circ p=g \circ q$. Then one of the following two cases holds:

(a) $p(z)=L \circ q(z)$ where $L$ is a linear polynomial.

(b) $p(z)=(r(z))^{2}+a$ and $q=b(r(z)+c)^{2}+d$, where $a, b, c, d$ are complex numbers.

The above type of results were first investigated by I.N. Baker and F. Gross in [1] and then L. Flatto in [5]. Finally, S.A. Lysenko in [8] gives an algebraic necessary and sufficient condition for the existence of meromorphic $f$ and $g$ satisfy $f \circ p=g \circ q$.

The proof of Theorem 3 is based on a method developed by S.A. Lysenko in [8] which depends on a fundamental result of local holomorphic dynamics. 


\section{Local holomorphic dynamics.}

Let $X$ be a Riemann surface and let $f:(X, a) \rightarrow(X, a)$ denote a mapping defined in some neighbourhood of a point $a$ on $X$ with $f(a)=a$. A germ of a mapping $f:(X, a) \rightarrow(X, a)$ is defined to be the equivalent class of all mappings which coincide with $f$ in some neighbourhood of $a$ and it is denoted by $[f]$. We say that $f$ is conformal at $a$ if $f$ is analytic in some neighbourhood of $a$ and $f^{\prime}(a) \neq 0$. In this case $f$ will have an inverse $f^{-1}$ in a neighbourhood of $a$. Let $\Gamma(X, a)$ be the set of all germs of conformal mapping $(X, a) \rightarrow(X, a)$. We define $[f] \circ[g]$ by $[f \circ g]$. Note that if $[f]=\left[f_{1}\right]$, then $f \equiv f_{1}$ on any region for which both $f$ and $f_{1}$ are analytic. Hence, the binary operation $\circ$ is well-defined. Clearly, the inverse of $[f]$ under $\circ$ is $\left[f^{-1}\right]$. Therefore, $(\Gamma(X, a), \circ)$ is a group. Note that two germs in $(\Gamma(X, a), \circ)$ are the same if they have the same Talyor series expansions about $a$. Therefore, from time to time, we shall simply denote the germ $[f]$ by its Talyor series.

For example, elements of $\Gamma\left(\mathbf{C P}^{\mathbf{1}}, \infty\right)$ are of the form $a_{1} z+a_{0}+\frac{a_{-1}}{z}+$ $\frac{a_{-2}}{z^{2}}+\cdots$ with $a_{1} \neq 0$. While elements of $\Gamma(\mathbf{C}, 0)$ are of the form $a_{1} z+$ $a_{2} z^{2}+a_{3} z^{3}+\cdots$ with $a_{1} \neq 0$.

We simply denote $\Gamma\left(\mathbf{C P}^{1}, \infty\right)$ by $\Gamma$.

Definition 3. Let $p$ be a nonconstant polynomial. Since $p^{-1}(\{\infty\})=\{\infty\}$, we can define a group $T_{p}=\{g \in \Gamma \mid p \circ g=p\}$. Then, it can be shown that $T_{p}$ is a cyclic subgroup of $\Gamma$ and its order equals to deg $p$.

Example 1. $T_{z^{n}}=\left\{\lambda z \mid \lambda^{n}=1\right\}$ and $T_{(z+1)^{m}}=\left\{\delta z+\delta-1 \mid \delta^{m}=1\right\}$.

$T_{p}$ is so-called a discrete invariant subgroup of $\Gamma$. In fact, we have the following definition.

Definition 4. A subgroup $G$ of $\Gamma$ is discrete invariant if there exists a nonconstant function $F$, meromorphic in a punctured neighbourhood of infinity in $\mathbf{C}$, such that $F(g(z))=F(z)$ for all $g \in G$.

In [11], A.A. Shcherbakov proved that if $G \subset \Gamma$ is discrete invariant, then $G$ is a solvable group.

We also need another important necessary condition for $G \subset \Gamma$ to be discrete. Define $\Gamma_{1}=\left\{g \in \Gamma \mid g=z+a_{0}+\frac{a_{-1}}{z}+\frac{a_{-2}}{z^{2}}+\cdots\right\}$ and $\Gamma_{0}=$ $\left\{g \in \Gamma \mid g=z+\frac{a_{-1}}{z}+\frac{a_{-2}}{z^{2}}+\cdots\right\}$. Clearly, $\Gamma_{1} / \Gamma_{0}$ is isomorphic to $(\mathbf{C},+)$.

Lemma 7 ([8]). Let $G \subset \Gamma, G_{1}=G \cap \Gamma_{1}$ and $G_{0}=G \cap \Gamma_{0}$. If $G$ is discrete invariant, then $G_{1} / G_{0}$ is isomorphic to a discrete subgroup of $(\mathbf{C},+)$.

Example 2. Let $f, g$ be nonconstant meromorphic functions and $p, q$ be nonconstant polynomials. Suppose that $F(z)=f(p(z))=g(q(z))$, then the group generated by $T_{p}$ and $T_{q}$, denoted by $\left[T_{p}, T_{q}\right]$, is a discrete invariant 
subgroup of $\Gamma$. Hence, $\left[T_{p}, T_{q}\right]$ is solvable. If we take $p(z)=z^{n}, q(z)=$ $(z+1)^{m}$ and $G=\left[T_{z^{n}}, T_{(z+1)^{m}}\right]$, then $G_{1} \subset\left\{T_{b}(z)=z+b \mid b \in \mathbf{C}\right\}$ and $G_{0}=\{z\}$. Now $G_{1} \cong G_{1} / G_{0}$ which is isomorphic to a discrete subgroup of $(\mathbf{C},+)$.

$T_{p}$ and $\left[T_{p}, T_{q}\right]$ are the main objects we shall study. The following two lemmas which were proved by using Galois Theory will be needed in the proof of Theorem 3 .

Lemma 8 ([8]). Let $p$ and $q$ be two nonconstant polynomials. Define $H_{p, q}$ $=\left\{\sigma \in T_{p} \mid \rho \sigma=\sigma \rho\right.$ for all $\left.\rho \in T_{q}\right\}$. Then $H_{p, q}=T_{p_{1}}$, where $p_{1}$ is a right factor of $p$.

Lemma $9([8])$. If $\left[T_{p}, T_{q}\right]$ is finite, then there exist two nonconstant rational functions $R_{1}, R_{2}$ such that $R_{1} \circ p(z)=R_{2} \circ q(z)$.

If $\left[T_{p}, T_{q}\right]$ is infinite, then $\left[T_{p}, T_{q}\right]$ must be non-Abelian as both $T_{p}$ and $T_{q}$ are cyclic. Moreover, if $\left[T_{p}, T_{q}\right]$ is also solvable, then we can construct some groups that are isomorphic to $\left[T_{p}, T_{q}\right]$. These groups come from local holomorphic dynamics and are easier to deal with.

Definition 5. Let $w$ be a holomorphic vector field on $V \subset$ C. Associated with $w$, it is well known that there exists a unique local phase flow $g_{w}$ : $U \times V \rightarrow \mathbf{C}$ which is a solution of the Cauchy problem

$$
\frac{d}{d t} g_{w}(t, z)=w\left(g_{w}(t, z)\right), \quad g_{w}(0, z)=z,
$$

where $U \subset \Re$ is a sufficiently small neighbourhood of 0 . For brevity, we denote $g_{w}(t, z)$ by $g_{w}^{t}(z)$ the time-t transformation for the flow of the holomorphic vector field $w$. Moreover, we have the following important property:

$$
g_{w}^{t+s}(z)=g_{w}^{t}\left(g_{w}^{s}(z)\right),
$$

in the sense that if one side of (4) is defined, so is the other, and they are equal. If we extend the definition of $g_{w}^{t}(z)$ for all $t \in \mathbf{C}$, then $g_{w}^{t}(z)$ (possibly divergent) will be a formal solution of Equation (3), which will be denoted as $\widehat{g_{w}^{t}}(z)$.

Definition 6. If $f: V \rightarrow W$ is a bijective conformal mapping, then the forward image $f_{*} w$ of the vector field $w$ on $V$ is defined as

$$
\left(f_{*} w\right)(z)=f^{\prime}\left(f^{-1}(z)\right) \times w\left(f^{-1}(z)\right),
$$

for all $z \in W$.

Let $k$ be a natural number. We denote by $g_{z^{k+1}}^{t}$ the time- $t$ transformation for the flow of the holomorphic vector field $z^{k+1} \frac{\partial}{\partial z}$. Express $g_{z^{k+1}}^{t}$ as $a_{0}(t)+a_{1}(t) z+a_{2}(t) z^{2}+\cdots$ and substitute it into Equation (3). Comparing the coefficient of the constant term, we have $a_{0}^{\prime}(t)=a_{0}^{k+1}(t), \quad a_{0}(0)=0$. 
Hence, $a_{0}(t) \equiv 0$ on some neighbourhood of zero. By repeating this process, it is easy to check that $g_{z^{k+1}}^{t}(z)=z+t z^{k+1}+\cdots$. Therefore, for each sufficiently small real $t, g_{z^{k+1}}^{z^{z}}(z)$ is conformal in some neighbourhood of zero with $g_{z^{k+1}}^{t}(0)=0$. Note that for complex number $|t|<1$, we have $g_{z^{2}}^{t}(z)=z+t z^{2}+t^{2} z^{3}+t^{3} z^{4}+\cdots$ is conformal in some neighbourhood of zero.

Now, we consider the set of germs

$$
G(k)=\left\{\lambda g_{z^{k+1}}^{t}:(\mathbf{C}, 0) \rightarrow(\mathbf{C}, 0) \mid \lambda \in \mathbf{C}^{*}=\mathbf{C}-\{0\}, t \in \mathbf{C}\right\} .
$$

We shall show that $G(k)$ under composition is a group. For brevity, denote $\lambda g_{z^{k+1}}^{t}$ by $(\lambda, t)$. For any $\mu \in \mathbf{C}^{*}$, let $\mu(z)=\mu z$, it is easy to check that $\mu^{-1} \circ g_{\mu_{*} w}^{t} \circ \mu$ satisfies condition (3) and hence $g_{\mu_{*} w}^{t} \circ \mu=\mu \circ g_{w}^{t}$. Similarly, we have $g_{z^{k+1}}^{t}=g_{\mu_{*} z^{k+1}}^{\mu^{-k} t}$. Now,

$$
g_{z^{k+1}}^{t} \circ \mu=g_{\mu_{*} z^{k+1}}^{\mu^{-k} t} \circ \mu=\mu \circ g_{z^{k+1}}^{\mu^{-k} t} .
$$

(4) and (5) imply that $G(k)$ is a group under composition. From (4) and (5), the multiplication table for $G(k)$ has the following form:

$$
(\lambda, t) \times(\mu, s)=\left(\lambda \mu, t \mu^{-k}+s\right) .
$$

With the above formula, it is easy to prove that the subgroup $C(k)=\{\lambda z=$ $\left.\lambda g_{z^{k+1}}^{0} \in G(k) \mid \lambda^{k}=1\right\}$ is the center of $G(k)$ (i.e., set of element commutes with all elements of $G(k))$.

Definition 7. Let $G$ and $G_{1}$ be two groups of germs of conformal mappings $(\mathbf{C}, 0) \rightarrow(\mathbf{C}, 0) . G$ and $G_{1}$ is said to be formally equivalent if there exists an isomorphism $K: G \rightarrow G_{1}$ and a formal series $\widehat{h}$ whose constant term is zero and the linear term is nonzero, such that for any $f \in G$,

$$
\widehat{h^{-1}} \circ f \circ \widehat{h}=\widehat{K f} \text {. }
$$

The hat over a symbol stands for the corresponding formal series.

Now, we can state the main lemma as follows.

Lemma $10([3])$. A finitely generated non-Abelian solvable group of all germs of conformal mapping $(\mathbf{C}, 0) \rightarrow(\mathbf{C}, 0)$ is formally equivalent to a finitely generated subgroup of $G(k)$ for some $k$.

Remark 2. Let $J(z)=1 / z$ and $G$ be a subgroup of $\Gamma\left(\mathbf{C P}^{\mathbf{1}}, \infty\right)$. Then $J^{-1} G J=\left\{J^{-1} \circ g \circ J \mid g \in G\right\}$ is a subgroup of $\Gamma(\mathbf{C}, 0)$. Clearly $G$ and $J^{-1} G J$ are isomorphic and from now on, we shall identify $G$ with $J^{-1} G J$ frequently. For example, $T_{z^{n}}$ is identified with $J^{-1} T_{z^{n}} J=\left\{\lambda z \mid \lambda^{n}=1\right\}=$ $\left\{\lambda g_{z^{2}}^{0} \mid \lambda^{n}=1\right\}$ and $T_{(z+1)^{m}}$ is identified with $J^{-1} T_{(z+1)^{m}} J=\{\delta z+\delta(\delta-$ 1) $\left.z^{2}+\delta(\delta-1)^{2} z^{3}+\delta(\delta-1)^{3} z^{4}+\cdots \mid \delta^{m}=1\right\}=\left\{\delta g_{z^{2}}^{\delta-1} \mid \delta^{m}=1\right\}$. 


\section{Proof of Theorem 3.}

Let $F(z)=f(p(z))=g(q(z))$. From Example 2, we know that $\left[T_{p}, T_{q}\right]$ is solvable. We shall consider two cases: i) $\left[T_{p}, T_{q}\right]$ is finite and ii) $\left[T_{p}, T_{q}\right]$ is infinite.

Suppose that $\left[T_{p}, T_{q}\right]$ is finite, then by Lemma 9 , there exist two nonconstant rational functions $R_{1}, R_{2}$ such that $R_{1} \circ p(z)=R_{2} \circ q(z)$. Express $R_{i}$ as $\frac{P_{i}}{Q_{i}}$, where $P_{i}$ and $Q_{i}$ are polynomials and do not have any common zero. Without loss of generality, we may assume that $P_{1}$ is nonconstant. Since $P_{i}$ and $Q_{i}$ do not have any common zero, we have $F_{1}=P_{1}(p(z))=A P_{2}(q(z))$ for some nonzero constant $A$. By Lemma 2 , there exists a nonconstant entire function $F_{2}$, which is the least generalized common left multiple of $p$ and $q$, such that $F_{2} \leq F_{1}$ and $F_{2} \leq F$. From $F_{2} \leq F_{1}$, it follows that $F_{2}$ is a polynomial and hence $F_{2} \mid F_{1}$ and $F_{2} \mid F$. Now, we can let $F_{2}=h \circ p=k \circ q$ for some polynomials $h, k$. Note that $F_{2} \mid F$ which implies $h \mid f$. Since $f$ is prime and transcendental, $h$ must be linear. Therefore, $p=h^{-1} \circ k \circ q$, where $h^{-1} \circ k$ is linear because $p$ is prime and $q$ is nonlinear. So, we are done for case i).

If $\left[T_{p}, T_{q}\right]$ is infinite, then it is non-Abelian as both $T_{p}, T_{q}$ are finite order cyclic groups. Since $\left[T_{p}, T_{q}\right]$ is also solvable, it follows from Lemma 10 that $\left[T_{p}, T_{q}\right]$ is formally equivalent to a subgroup of $G(k)$ for some natural number $k$. Let $\mathrm{d}=\operatorname{lcm}(\mathrm{n}, \mathrm{m})$ where $\mathrm{n}=\operatorname{deg} p$ and $\mathrm{m}=\operatorname{deg} q$. Let $\lambda g_{z^{k+1}}^{t}$ and $\mu g_{z^{k+1}}^{s}$ be the generators of $T_{p}$ and $T_{q}$ respectively. From the multiplication table of $G(k), \lambda^{n}=1$ and $\mu^{m}=1$. Hence, all elements of $\left[T_{p}, T_{q}\right]$ are in $G_{d}(k)=\left\{\lambda g_{z^{k+1}}^{t} \in G(k) \mid \lambda^{d}=1\right\}$. Therefore, $\left[T_{p}, T_{q}\right]$ is actually formally equivalent to a subgroup of $G_{d}(k)$.

By Lemma 8 and the fact that $p$ is prime, $H_{p, q}=T_{p}$ or $T_{i d}$. If $H_{p, q}=T_{p}$, then $\left[T_{p}, T_{q}\right]$ must be abelian which is impossible. So, we have $H_{p, q}=T_{i d}=$ $\{z\}$. It is easy to check that if $h \in G_{k}(k)$ is an element of finite order, then $h \in C(k)$. Hence, $T_{p} \cap G_{k}(k) \subset C(k)$. Note that $C(k)$ is the center of $G(k)$ and so $T_{p} \cap G_{k}(k) \subset H_{p, q}=\{z\}$. Now, we claim that $\mathrm{g}=\operatorname{gcd}(\mathrm{n}, \mathrm{k})=1$. Let $(\lambda, t)$ be a generator of $T_{p}$. Then, it is very easy to check that $(\lambda, t)^{\frac{n}{g}}$ is an element of $T_{p} \cap G_{k}(k)$. Therefore, $(\lambda, t)^{\frac{n}{g}}=(1,0)$ and hence $\frac{n}{g}=n$. We get $\mathrm{g}=\operatorname{gcd}(\mathrm{n}, \mathrm{k})=1$.

We first consider the case that $q$ is prime. Then, we also have $\operatorname{gcd}(\mathrm{m}, \mathrm{k})=$ 1. So, if $\mathrm{d}=\operatorname{lcm}(\mathrm{n}, \mathrm{m})$, then $\operatorname{gcd}(\mathrm{d}, \mathrm{k})=1$. We define a map $f: G_{d}(k) \rightarrow$ $G_{d}(1)$ by $f\left(\lambda g_{z^{k+1}}^{t}\right)=\lambda^{k} g_{z^{2}}^{t}$. Clearly, $f$ is a group homorphism and surjective. The condition that $\operatorname{gcd}(\mathrm{d}, \mathrm{k})=1$ implies that $f$ is also injective. Therefore $\left[T_{p}, T_{q}\right]$ is isomorphic to a subgroup of $G_{d}(1)$. 
Let $\lambda g_{z^{2}}^{t}$ and $\delta g_{z^{2}}^{s}$ be the elements of $G_{d}(1)$ corresponding to generators of $T_{p}$ and $T_{q}$ respectively. Note that

$$
(1,0)=\mathrm{id}=\lambda g_{z^{2}}^{t} \circ \lambda g_{z^{2}}^{t} \cdots \circ \lambda g_{z^{2}}^{t}(n \text { times })=\left(\lambda^{n}, t\left(\lambda^{-(n-1)}+\cdots+\lambda^{-1}+1\right)\right) .
$$

So, $\lambda$ (respectively $\delta$ ) is a primitive $n$th root of unity (respectively a primitive $m$ th root of unity).

By choosing a suitable number $r$, we have $(1, r) \times(\lambda, t) \times(1,-r)=(\lambda, 0)$. Therefore, with this conjugation, we may assume $t=0$ and this implies that $s \neq 0$, for otherwise $\left[T_{p}, T_{q}\right]$ will be abelian. By using the automorphism $\lambda g_{z^{2}}^{t} \rightarrow \lambda g_{z^{2}}^{c t}(c \neq 0)$ of $G_{d}(1)$, we may also assume that $s=\delta-1$. Hence the generators are of the form $\lambda g_{z^{2}}^{0}$ and $\delta g_{z^{2}}^{\delta-1}$. From Remark 2, we know that they generate $T_{z^{n}}$ and $T_{(z+1)^{m}}$ respectively. Therefore $\left[T_{p}, T_{q}\right]$ is isomorphic to $G=\left[T_{z^{n}}, T_{(z+1)^{m}}\right]$. From Example $2, G_{1} \cong\left(G_{1} / G_{0}\right) \cong$ $\left(\left[T_{p}, T_{q}\right] \cap \Gamma_{1}\right) /\left(\left[T_{p}, T_{q}\right] \cap \Gamma_{0}\right)$ which is isomorphic to a discrete subgroup of $(\mathbf{C},+)$ by Lemma 7 .

Suppose $T_{b} \in G_{1}$, then $T_{\delta b}$ is also in $G_{1}$. It is because $z+\delta b=(\delta z+$ $\delta-1) \circ(z+b) \circ\left(\delta^{-1} z+\delta^{-1}-1\right)$. Similarly, $T_{\lambda b} \in G_{1}$ and hence $T_{\epsilon b} \in G_{1}$, where $\epsilon$ is a $d$ th root of unity with $d=\operatorname{lcm}(\mathrm{n}, \mathrm{m})$. Since $G_{1}$ is isomorphic to a nontrivial discrete subgroup of $(\mathbf{C},+)$, it is easy to show that either $G_{1}=\left\{T_{n a} \mid n \in \mathbf{Z}\right\}$ or $G_{1}=\left\{T_{n b+m c} \mid n, m \in \mathbf{Z}\right\}$ for some $a, b, c \in \mathbf{C}$ and $b / c$ being irrational (see [9], p. 63). We consider the first case: $T_{a} \in G_{1}$, which implies $T_{\epsilon^{2} a+a} \in G_{1}$. Hence, $T_{2 a \cos \frac{2 \pi}{d}}=T_{\epsilon^{-1} \times\left(\epsilon^{2} a+a\right)} \in G_{1}$. Thus, 2 $\cos \frac{2 \pi}{d}$ is some integer which can only be $0, \pm 1$ or \pm 2 . So, it follows that $d \in\{2,3,4,6\}$. With similar argrument, we can have the same conclusion for the second case.

If $n=m=3,4,6$, then it follows from Lemma 6 that $p=L \circ q$, where $L$ is linear. Hence, $\left[T_{p}, T_{q}\right]=T_{p}$ is finite, which is a contradiction.

If $n=m=2$, without loss of generality we may assume that $p(z)=z^{2}$ and $q(z)=(z+c)^{2}$. Then we have $F_{1}=\cos \sqrt{z} \circ p=\cos (\sqrt{z}-c) \circ q$. By Lemma 2, there exists a nonconstant entire function $F_{2}$, which is the least generalized common left multiple of $p$ and $q$, such that $F_{2} \leq F_{1}$ and $F_{2} \leq F$. Let $F_{2}=h \circ p=k \circ q$, it follows that $h \leq f$ and $h \leq \cos \sqrt{z}$. Thus $h$ is not periodic. By similar argrument used in the proof of Theorem 1, we have $h \mid f$. Since $f$ is prime, $h$ is linear or $h=L \circ f$ for some linear function $L$. $h$ is linear implies $p=h^{-1} \circ k \circ q$ which is impossible again. Therefore, $h=L \circ f$. Hence, $\cos \sqrt{z}$ has a prime transcendental right factor $f$. Write $\cos \sqrt{z}$ as $h_{1} \circ f$. Thus $\cos z=h_{1} \circ f\left(z^{2}\right)$. From Theorem 3.10 in [2], $f\left(z^{2}\right)=\cos \frac{z}{n}$ which implies $f(z)=\cos \frac{\sqrt{z}}{n}$. This is impossible as $\cos \frac{\sqrt{z}}{n}$ is not a prime function.

Now, we can assume that $n \neq m$ and hence $d \neq 2,3 . d=4$ implies that one of $n, m$ equals to 2 . We may assume without loss of generality that $n=2$ and $p=z^{2}, q(z)=z^{4}+a_{3} z^{3}+a_{2} z^{2}+a_{1} z$. Since $f(p(z))=f(p(-z))$, 
$g(q(z))=g(q(-z))$, and because $q$ is prime, Lemma 6 implies that $q(z)=$ $L \circ q(-z)$. Note that $L$ is linear, then $a_{3}=a_{1}=0$ and hence $q$ is not prime which is impossible. If $d=6, n$ can only be 2,3 or 6 . The case for $n=2$ can be treated similiar as above and the case $n=3,6$ are excluded from our considerations.

For general $q$, we can express $q$ as $q_{2} \circ q_{1}$ where $q_{1}$ is prime. From the above discussion, we have $f=g \circ q_{2} \circ L^{-1}$ and $p=L \circ q_{1}$. Thus, $f$ is prime implies that $q_{2}$ is linear and we are done.

\section{Further discussions.}

In Theorem 3, we assume that both the right factors $p, q$ have polynomial growth. We can also restrict the left factors $f, g$ to have comparable growth rate and ask the following question.

Problem (B). Let $f$ and $p$ be two prime entire functions and $p$ is a polynomial. Suppose that $F=f \circ p=g \circ q$ and both $f, g$ are transcendental. Are the two factorizations of $F$ equivalent?

This problem is closely related to Problem C below (proposed by C.C. Yang, see e.g., [7], p. 124), which remains unsolved for more than a decade.

Problem (C). Let $f$ be a pseudo-prime transcendental meromorphic function and $p$ be a polynomial of degree $\geq 2$. Must $f(p(z))$ be pseudo-prime?

If the answer to Problem $\mathrm{C}$ is positive, then the function $q$ in Problem $\mathrm{B}$ must be a polynomial and this reduces to the case handled in Theorem 3 . One may try to solve Problem $\mathrm{C}$ for the special case that $p(z)=z^{n}$, where $n$ is a prime number.

Similarly, we can ask:

Problem (D). Let $f$ be a pseudo-prime transcendental meromorphic function and $p$ a polynomial of degree $\geq 3$, which has no quadratic right factor. Must $p(f(z))$ be pseudo-prime?

In [12], G.D. Song and J. Huang proposed the above problem and solved it for the case that $p(z)=z^{n}$ with $n$ being an odd number. We proved in [10] that it is true if $f$ is not of the form $H \circ q$, where $H$ is an entire periodic function and $q$ is a polynomial. One may try to solve Problem $\mathrm{D}$ for $\operatorname{deg} \mathrm{p}$ is odd first.

Finally, we ask whether the answer of Problem A is yes if both $f$ and $g$ are assumed to be transcendental?

\section{References}

[1] I.N. Baker and F. Gross, On factorizing entire functions, Proc. London Math. Soc., 18(3) (1968), 69-76. 
[2] C.T. Chuang and C.C. Yang, Fix-points and factorization of meromorphic functions, World Scientific, Singapore, 1990.

[3] P.J. Elizarov, Yu.S. Il'yashenko, A.A. Shcherbakov and S.M. Voronian, Finitely generated groups of germs of one-dimensional conformal mappings and invariants for complex singular points of analytic foliations of the complex plane, Advances in Soviet Math., 14, Amer. Math. Soc., Providence, RI, (1993), 57-105.

[4] A. Eremenko and L.A. Rubel, The arithmetic of entire functions under composition, Advances in Mathematics, 124 (1996), 334-354.

[5] L. Flatto, A theorem on level curves of harmonic functions, J. London Math. Soc., 1 (1969), 470-472.

[6] W.H.J. Fuchs and G.D. Song, On a conjecture by M. Ozawa concerning factorization of entire functions, Ann. Acad. Sci. Fennicae Ser. A. I. Math., 10 (1985), 173-185.

[7] Y.Z. He and C.C. Yang, On pseudo-primality of the product of some pseudo-prime meromorphic functions, Analysis of one complex variable, edited by C.C. Yang, 113124, World Scientific, Singapore, 1985.

[8] S.A. Lysenko, On the functional equation $f(p(z))=g(q(z))$, where $p$ and $q$ are "generalized" polynomials and $f$ and $g$ are meromorphic functions, Izvestiya: Mathematics, 60 (1996), 89-110.

[9] T.T. Moh, Algebra, World Scientific, Singapore, 1992.

[10] T.W. Ng and C.C. Yang, Certain criteria on the existence of a transcendental entire common right factor, Analysis, 17, 387-393, 1997.

[11] A.A. Shcherbakov, On the denseness of orbits of the pseudogroup of conformal mappings and a generalization of the Huday-Verenov theorem, Vestnik Mosk. Gos. Univ. Ser. 1, Mat.-Mekh., 2 (1982), 10-15; English transl. in Moscow Univ. Math. Bull., 37 (1982).

[12] G.D. Song and J. Huang, On pseudo-primality of the n-th power of prime entire functions, Kodai Math. J., 10 (1987), 42-48.

Received July 29, 1998 and revised October 28, 1998. This research was partially supported by a UGC grant of Hong Kong (project no. HKUST710/96P).

Department of Pure Mathematics and Mathematical Statistics

University of CAmbridge

16 Mill Lane, Cambridge CB2 1SB

ENGLAND

E-mail address: ntw@dpmms.cam.ac.uk

Department of Mathematics

Hong Kong University of Science and Technology

Clear Water Bay

Kowloon, Hong Kong

CHINA

E-mail address: mayang@ust.hk 